\title{
Interband Multiphoton Absorption of Light in Narrow-Gap Crystals
}

\author{
V. R. Rasulov, R. Ya. Rasulov, I. M. Eshboltaev, M. X. Qo'chqorov
}

\section{ABSTRACT}

The spectral dependences of the coefficients of interband three-photon optical transitions for InSb and for some optical transitions are calculated and a numerical analysis of the coefficient of interband three-photon absorption of light is carried out, which taken into account the contribution to the three-photon absorption of optical transitions occurring during the simultaneous absorption of two photons in the Kane model.

Interband three-photon optical transitions in crystals of the InSb and type are classified, and the spectral dependence of some optical transitions is analyzed. The coefficient of linear-circular dichroism of interband threephoton absorption in a crystal in the Kane model is calculated.

Keywords: interband three-photon optical transitions, spectral dependence, light absorption coefficient, narrow-gap crystal.

Published Online: October 17, 2021

ISSN: $2684-4451$

DOI : 10.24018/ejphysics.2021.3.5.96

\section{R. Rasulov*}

Fergana State University, Uzbekistan.

(e-mail: r_rasulov51@mail.ru)

R. Ya. Rasulov

Fergana State University, Uzbekistan. I. M. Eshboltaev

Fergana State University, Uzbekistan. M. X. Qo'chqorov

Fergana State University, Uzbekistan.

*Corresponding Author

\section{INTRODUCTION}

The first works devoted to the research of two-photon interband transitions in crystals were carried out in the early 1960s, shortly after the appearance of lasers [1]-[3]. In calculating the matrix elements of two-photon transitions in crystals, perturbation theories were used in the field of an unpolarized electromagnetic wave [2], [3], where the two-band Kane model was used.

In [4]-[7], both theoretically and experimentally, linear-circular dichroism (LCD) of two- and three-photon absorption of light in crystals of cubic symmetry in the region of the center of the Brillouin zone was investigated. However, the question of spectral and temperature researches of multiphoton interband absorption of polarized light in narrow-gap crystals in the threeband Kane approximation remained open.

\section{MATRIX ELEMENTS OF THE THREE PHOtON INTERBAND OPTICAL TRANSITIONS}

Three-photon interband light absorption can be described by diagrams of type 3\{\} describes one photon absorption, $\left\{\right.$ describes the successive absorption of two photons, and $\frac{\xi}{\xi}$ describes the simultaneous absorption of two photons. Then three-photon optical transitions from the valence band $\left(\left|V_{l}, m_{l}\right\rangle\right)$ to the conduction band $\left(\left|c, m_{l}^{\prime}\right\rangle\right)$ generally have four types, which can be represented as the sum of the following optical transitions depending on the initial state of electrons participating in optical transitions:

a) the initial state of electrons is in the subband of heavy holes:

$$
\begin{aligned}
& \sum_{m_{l}, m_{l}^{\prime}= \pm 1 / 2, \pm 3 / 2}\left\{\left|V_{h h}, \pm 3 / 2\right\rangle \Rightarrow\left|V_{l}, m_{l}\right\rangle \rightarrow|c, \pm 1 / 2\rangle+\left|V_{h h}, \pm 3 / 2\right\rangle \rightarrow\left|V_{l}, m_{l}\right\rangle \rightarrow\left|V_{l}, m_{l}^{\prime}\right\rangle \rightarrow|c, \pm 1 / 2\rangle+\right. \\
& +\left|V_{h h}, \pm 3 / 2\right\rangle \rightarrow\left|\mathrm{c}, m_{l}\right\rangle \rightarrow\left|\mathrm{c}, m_{l}^{\prime}\right\rangle \rightarrow|c, \pm 1 / 2\rangle+\left|V_{h h}, \pm 3 / 2\right\rangle \rightarrow\left|\mathrm{SO}, m_{l}\right\rangle \rightarrow\left|\mathrm{SO}, m_{l}^{\prime}\right\rangle \rightarrow|c, \pm 1 / 2\rangle+ \\
& +\left|V_{h h}, \pm 3 / 2\right\rangle \rightarrow\left|V_{l}, m_{l}\right\rangle \rightarrow\left|\mathrm{c}, m_{l}^{\prime}\right\rangle \rightarrow|c, \pm 1 / 2\rangle+\left|V_{h h}, \pm 3 / 2\right\rangle \rightarrow\left|\mathrm{c}, m_{l}\right\rangle \rightarrow\left|V_{l}, m_{l}^{\prime}\right\rangle \rightarrow|c, \pm 1 / 2\rangle+ \\
& +\left|V_{h h}, \pm 3 / 2\right\rangle \rightarrow\left|V_{l}, m_{l}\right\rangle \rightarrow\left|\mathrm{SO}, m_{l}^{\prime}\right\rangle \rightarrow|c, \pm 1 / 2\rangle+\left|V_{h h}, \pm 3 / 2\right\rangle \rightarrow\left|S O, m_{l}\right\rangle \rightarrow\left|V_{l}, m_{l}^{\prime}\right\rangle \rightarrow|c, \pm 1 / 2\rangle+ \\
& \left.+\left|V_{h h}, \pm 3 / 2\right\rangle \rightarrow\left|\mathrm{c}, m_{l}\right\rangle \rightarrow\left|S O, m_{l}^{\prime}\right\rangle \rightarrow|c, \pm 1 / 2\rangle+\left|V_{h h}, \pm 3 / 2\right\rangle \rightarrow\left|\mathrm{SO}, m_{l}\right\rangle \rightarrow\left|c, m_{l}^{\prime}\right\rangle \rightarrow|c, \pm 1 / 2\rangle\right\},
\end{aligned}
$$

b) the initial state of electrons is in the subband of heavy holes: 


$$
\begin{aligned}
& \sum_{m_{l}, m_{l}^{\prime}= \pm 1 / 2, \pm 3 / 2}\left\{\left|V_{h h}, \mp 3 / 2\right\rangle \Rightarrow\left|V_{l}, m_{l}\right\rangle \rightarrow|c, \pm 1 / 2\rangle+\left|V_{h h}, \mp 3 / 2\right\rangle \rightarrow\left|V_{l}, m_{l}\right\rangle \rightarrow\left|V_{l}, m_{l}^{\prime}\right\rangle \rightarrow|c, \pm 1 / 2\rangle+\right. \\
& +\left|V_{h h}, \mp 3 / 2\right\rangle \rightarrow\left|\mathrm{c}, m_{l}\right\rangle \rightarrow\left|\mathrm{c}, m_{l}^{\prime}\right\rangle \rightarrow|c, \pm 1 / 2\rangle+\left|V_{h h}, \mp 3 / 2\right\rangle \rightarrow\left|\mathrm{SO}, m_{l}\right\rangle \rightarrow\left|\mathrm{SO}, m_{l}^{\prime}\right\rangle \rightarrow|c, \pm 1 / 2\rangle+ \\
& +\left|V_{h h}, \mp 3 / 2\right\rangle \rightarrow\left|V_{l}, m_{l}\right\rangle \rightarrow\left|\mathrm{c}, m_{l}^{\prime}\right\rangle \rightarrow|c, \pm 1 / 2\rangle+\left|V_{h h}, \mp 3 / 2\right\rangle \rightarrow\left|\mathrm{c}, m_{l}\right\rangle \rightarrow\left|V_{l}, m_{l}^{\prime}\right\rangle \rightarrow|c, \pm 1 / 2\rangle+ \\
& +\left|V_{h h}, \mp 3 / 2\right\rangle \rightarrow\left|V_{l}, m_{l}\right\rangle \rightarrow\left|\mathrm{SO}, m_{l}^{\prime}\right\rangle \rightarrow|c, \pm 1 / 2\rangle+\left|V_{h h}, \mp 3 / 2\right\rangle \rightarrow\left|S O, m_{l}\right\rangle \rightarrow\left|V_{l}, m_{l}^{\prime}\right\rangle \rightarrow|c, \pm 1 / 2\rangle+ \\
& \left.+\left|V_{h h}, \mp 3 / 2\right\rangle \rightarrow\left|\mathrm{c}, m_{l}\right\rangle \rightarrow\left|S O, m_{l}^{\prime}\right\rangle \rightarrow|c, \pm 1 / 2\rangle+\left|V_{h h}, \mp 3 / 2\right\rangle \rightarrow\left|\mathrm{SO}, m_{l}\right\rangle \rightarrow\left|c, m_{l}^{\prime}\right\rangle \rightarrow|c, \pm 1 / 2\rangle\right\} ;
\end{aligned}
$$

c) the initial state of electrons is in the subband of light holes:

$$
\begin{aligned}
& \sum_{m_{l}, m_{l}^{\prime}= \pm 1 / 2, \pm 3 / 2}\left\{\left|V_{l h}, \pm 1 / 2\right\rangle " \Rightarrow "\left|V_{l}, m_{l}\right\rangle " \rightarrow "|c, \pm 1 / 2\rangle+\left|V_{l h}, \pm 1 / 2\right\rangle \rightarrow\left|V_{l}, m_{l}\right\rangle \rightarrow\left|V_{l}, m_{l}^{\prime}\right\rangle \rightarrow|c, \pm 1 / 2\rangle+\right. \\
& +\left|V_{l h}, \pm 1 / 2\right\rangle \rightarrow\left|\mathrm{c}, m_{l}\right\rangle \rightarrow\left|\mathrm{c}, m_{l}^{\prime}\right\rangle \rightarrow|c, \pm 1 / 2\rangle+\left|V_{l h}, \pm 1 / 2\right\rangle \rightarrow\left|\mathrm{SO}, m_{l}\right\rangle \rightarrow\left|\mathrm{SO}, m_{l}^{\prime}\right\rangle \rightarrow|c, \pm 1 / 2\rangle+ \\
& +\left|V_{l h}, \pm 1 / 2\right\rangle \rightarrow\left|V_{l}, m_{l}\right\rangle \rightarrow\left|\mathrm{c}, m_{l}^{\prime}\right\rangle \rightarrow|c, \pm 1 / 2\rangle+\left|V_{l h}, \pm 1 / 2\right\rangle \rightarrow\left|\mathrm{c}, m_{l}\right\rangle \rightarrow\left|V_{l}, m_{l}^{\prime}\right\rangle \rightarrow|c, \pm 1 / 2\rangle+ \\
& +\left|V_{l h}, \pm 13 / 2\right\rangle \rightarrow\left|V_{l}, m_{l}\right\rangle \rightarrow\left|\mathrm{SO}, m_{l}^{\prime}\right\rangle \rightarrow|c, \pm 1 / 2\rangle+\left|V_{l h}, \pm 1 / 2\right\rangle \rightarrow\left|S O, m_{l}\right\rangle \rightarrow\left|V_{l}, m_{l}^{\prime}\right\rangle \rightarrow|c, \pm 1 / 2\rangle+ \\
& \left.+\left|V_{l h}, \pm 1 / 2\right\rangle \rightarrow\left|\mathrm{c}, m_{l}\right\rangle \rightarrow\left|S O, m_{l}^{\prime}\right\rangle \rightarrow|c, \pm 1 / 2\rangle+\left|V_{l h}, \pm 1 / 2\right\rangle \rightarrow\left|\mathrm{SO}, m_{l}\right\rangle \rightarrow\left|c, m_{l}^{\prime}\right\rangle \rightarrow|c, \pm 1 / 2\rangle\right\},
\end{aligned}
$$

d) the initial state of electrons is in the subband of light holes:

$$
\begin{aligned}
& \sum_{m_{l}, m_{l}^{\prime}= \pm 1 / 2, \pm 3 / 2}\left\{\left|V_{l h}, \mp 1 / 2\right\rangle \Rightarrow\left|V_{l}, m_{l}\right\rangle \rightarrow|c, \pm 1 / 2\rangle+\left|V_{l h}, \mp 1 / 2\right\rangle \rightarrow\left|V_{l}, m_{l}\right\rangle \rightarrow\left|V_{l}, m_{l}^{\prime}\right\rangle \rightarrow|c, \pm 1 / 2\rangle+\right. \\
& +\left|V_{l h}, \mp 1 / 2\right\rangle \rightarrow\left|\mathrm{c}, m_{l}\right\rangle \rightarrow\left|\mathrm{c}, m_{l}^{\prime}\right\rangle \rightarrow|c, \pm 1 / 2\rangle+\left|V_{l h}, \mp 1 / 2\right\rangle \rightarrow\left|\mathrm{SO}, m_{l}\right\rangle \rightarrow\left|\mathrm{SO}, m_{l}^{\prime}\right\rangle \rightarrow|c, \pm 1 / 2\rangle+ \\
& +\left|V_{l h}, \mp 1 / 2\right\rangle \rightarrow\left|V_{l}, m_{l}\right\rangle \rightarrow\left|\mathrm{c}, m_{l}^{\prime}\right\rangle \rightarrow|c, \pm 1 / 2\rangle+\left|V_{l h}, \mp 1 / 2\right\rangle \rightarrow\left|\mathrm{c}, m_{l}\right\rangle \rightarrow\left|V_{l}, m_{l}^{\prime}\right\rangle \rightarrow|c, \pm 1 / 2\rangle+ \\
& +\left|V_{l h}, \mp 13 / 2\right\rangle \rightarrow\left|V_{l}, m_{l}\right\rangle \rightarrow\left|\mathrm{SO}, m_{l}^{\prime}\right\rangle \rightarrow|c, \pm 1 / 2\rangle+\left|V_{l h}, \mp 1 / 2\right\rangle \rightarrow\left|S O, m_{l}\right\rangle \rightarrow\left|V_{l}, m_{l}^{\prime}\right\rangle \rightarrow|c, \pm 1 / 2\rangle+ \\
& \left.+\left|V_{l h}, \mp 1 / 2\right\rangle \rightarrow\left|\mathrm{c}, m_{l}\right\rangle \rightarrow\left|S O, m_{l}^{\prime}\right\rangle \rightarrow|c, \pm 1 / 2\rangle+\left|V_{l h}, \mp 1 / 2\right\rangle \rightarrow\left|\mathrm{SO}, m_{l}\right\rangle \rightarrow\left|c, m_{l}^{\prime}\right\rangle \rightarrow|c, \pm 1 / 2\rangle\right\} ;
\end{aligned}
$$

e) the initial states of electrons are in the spin-split band:

$$
\begin{aligned}
& \sum_{m_{l}, m_{i}^{\prime} \pm 1 / 2, \pm 3 / 2}\left\{|S O, \pm 1 / 2\rangle \Rightarrow\left|V_{l}, m_{l}\right\rangle \rightarrow|c, \pm 1 / 2\rangle+|S O, \pm 1 / 2\rangle \rightarrow\left|V_{l}, m_{l}\right\rangle \rightarrow\left|V_{l}, m_{l}^{\prime}\right\rangle \rightarrow|c, \pm 1 / 2\rangle+\right. \\
& +|S O, \pm 1 / 2\rangle \rightarrow\left|\mathrm{c}, m_{l}\right\rangle \rightarrow\left|\mathrm{c}, m_{l}^{\prime}\right\rangle \rightarrow|c, \pm 1 / 2\rangle+|S O, \pm 1 / 2\rangle \rightarrow\left|\mathrm{SO}, m_{l}\right\rangle \rightarrow\left|\mathrm{SO}, m_{l}^{\prime}\right\rangle \rightarrow|c, \pm 1 / 2\rangle+ \\
& +|S O, \pm 1 / 2\rangle \rightarrow\left|V_{l}, m_{l}\right\rangle \rightarrow\left|\mathrm{c}, m_{l}^{\prime}\right\rangle \rightarrow|c, \pm 1 / 2\rangle+|S O, \pm 1 / 2\rangle \rightarrow\left|\mathrm{c}, m_{l}\right\rangle \rightarrow\left|V_{l}, m_{l}^{\prime}\right\rangle \rightarrow|c, \pm 1 / 2\rangle+ \\
& +|S O, \pm 13 / 2\rangle \rightarrow\left|V_{l}, m_{l}\right\rangle \rightarrow\left|\mathrm{SO}, m_{l}^{\prime}\right\rangle \rightarrow|c, \pm 1 / 2\rangle+|S O, \pm 1 / 2\rangle \rightarrow\left|S O, m_{l}\right\rangle \rightarrow\left|V_{l}, m_{l}^{\prime}\right\rangle \rightarrow|c, \pm 1 / 2\rangle+ \\
& \left.+|S O, \pm 1 / 2\rangle \rightarrow\left|\mathrm{c}, m_{l}\right\rangle \rightarrow\left|S O, m_{l}^{\prime}\right\rangle \rightarrow|c, \pm 1 / 2\rangle+|S O, \pm 1 / 2\rangle \rightarrow\left|\mathrm{SO}, m_{l}\right\rangle \rightarrow\left|c, m_{l}^{\prime}\right\rangle \rightarrow|c, \pm 1 / 2\rangle\right\},
\end{aligned}
$$

f) the initial states of electrons are in the spin-split band:

$$
\begin{aligned}
& \sum_{m_{l}, m_{l}^{\prime}= \pm 1 / 2 \pm 3 / 2}\left\{|S O, \mp 1 / 2\rangle \Rightarrow\left|V_{l}, m_{l}\right\rangle \rightarrow|c, \pm 1 / 2\rangle+|S O, \mp 1 / 2\rangle \rightarrow\left|V_{l}, m_{l}\right\rangle \rightarrow\left|V_{l}, m_{l}^{\prime}\right\rangle \rightarrow|c, \pm 1 / 2\rangle+\right. \\
& +|\mathrm{SO}, \mp 1 / 2\rangle \rightarrow\left|\mathrm{c}, m_{l}\right\rangle \rightarrow\left|\mathrm{c}, m_{l}^{\prime}\right\rangle \rightarrow|c, \pm 1 / 2\rangle+|S O, \mp 1 / 2\rangle \rightarrow\left|\mathrm{SO}, m_{l}\right\rangle \rightarrow\left|\mathrm{SO}, m_{l}^{\prime}\right\rangle \rightarrow|c, \pm 1 / 2\rangle+ \\
& +|\mathrm{SO}, \mp 1 / 2\rangle \rightarrow\left|V_{l}, m_{l}\right\rangle \rightarrow\left|\mathrm{c}, m_{l}^{\prime}\right\rangle \rightarrow|c, \pm 1 / 2\rangle+|S O, \mp 1 / 2\rangle \rightarrow\left|\mathrm{c}, m_{l}\right\rangle \rightarrow\left|V_{l}, m_{l}^{\prime}\right\rangle \rightarrow|c, \pm 1 / 2\rangle+ \\
& +|\mathrm{SO}, \mp 1 / 2\rangle \rightarrow\left|V_{l}, m_{l}\right\rangle \rightarrow\left|\mathrm{SO}, m_{l}^{\prime}\right\rangle \rightarrow|c, \pm 1 / 2\rangle+|S O, \mp 1 / 2\rangle \rightarrow\left|S O, m_{l}\right\rangle \rightarrow\left|V_{l}, m_{l}^{\prime}\right\rangle \rightarrow|c, \pm 1 / 2\rangle+ \\
& \left.+|S O, \mp 1 / 2\rangle \rightarrow\left|\mathrm{c}, m_{l}\right\rangle \rightarrow\left|S O, m_{l}^{\prime}\right\rangle \rightarrow|c, \pm 1 / 2\rangle+|S O, \mp 1 / 2\rangle \rightarrow\left|\mathrm{SO}, m_{l}\right\rangle \rightarrow\left|c, m_{l}^{\prime}\right\rangle \rightarrow|c, \pm 1 / 2\rangle\right\},
\end{aligned}
$$

where each component differs from each other in the order of virtual states, which can appear both in the subbands of the valence band $\left(\left|V_{l}, m_{l}\right\rangle\right)$ and in the conduction band $\left(\left|\mathrm{c}, m_{l}^{\prime}\right\rangle\right)$ or in the spin-split band $\left(\left|S O, m_{l}^{\prime}\right\rangle\right), m_{l}, m_{l}^{\prime}= \pm 3 / 2$ for subbands of heavy holes, $m_{l}, m_{l}^{\prime}= \pm 1 / 2$ for subbands of light holes, conduction bands and spin-split-off bands, $m_{l}$ or $m_{l}^{\prime}$ the eigenvalue of the total momentum operator. $\left|A, m_{a}\right\rangle \Rightarrow\left|B, m_{b}\right\rangle$ characterizes the optical transition from state $\left|A, m_{a}\right\rangle$ to $\left|B, m_{b}\right\rangle$, occurring by the simultaneous absorption of two photons (see, for example, [5-7]), $\left|A, m_{a}\right\rangle \rightarrow\left|B, m_{b}\right\rangle$ characterizes a one-photon optical transition from the $\left|A, m_{a}\right\rangle$ to $\left|B, m_{b}\right\rangle$ state.

Note that some of the above matrix elements may turn out to be equal to zero in the zero, linear, and quadratic approximation in the wave vector in the energy spectrum (in the effective Hamiltonian). 
In what follows, we choose the following Luttinger Cohn basis functions [8]. Then the effective Hamiltonian in the above sequence of basic functions takes the form:

where each component differs from each other by the order of the band parameters [8]. In particular, the dimensionless Luttinger constants $\gamma_{1}, \gamma_{2}, \gamma_{3}$ are related to the band parameters $A, B, D$ as $\frac{\hbar^{2}}{2 m_{0}} \gamma_{1}=A, \frac{\hbar^{2}}{2 m_{0}} \gamma_{2}=\frac{B}{2}, \frac{\hbar^{2}}{2 m_{0}} \gamma_{3}=\frac{D}{2 \sqrt{3}}$, the numerical values of which are given in [9]. Then the energy spectrum of light and heavy holes takes the form $E_{l h}(\vec{k})=E_{v}-(A+B) k^{2}, E_{h h}(\vec{k})=E_{v}-(A-B) k^{2}$, where the effective masses of light and heavy holes do not depend on the direction of the wave vector and are determined by the relations: $\frac{\hbar^{2}}{2 m_{l h}}=A+B=\frac{\hbar^{2}}{2 m_{0}}\left(\gamma_{1}+2 \gamma_{2}\right)$, $\frac{\hbar^{2}}{2 m_{h h}}=A-B=\frac{\hbar^{2}}{2 m_{0}}\left(\gamma_{1}-2 \gamma_{2}\right)$. In this case, the matrix elements of the pulse operator are determined by $\vec{e} \cdot \vec{\nabla} H(\vec{k})$, where $\dot{e}$ is the vector of the polarization of the light.

Note that there are 16 types of optical transitions that differ from each other in virtual states. Therefore, below we will consider individual optical transitions. For example, the matrix element of an optical transition described by the following diagrams is determined by the relation, and for transitions of the type $|V,-3 / 2\rangle \rightarrow|\mathrm{V},-3 / 2\rangle \rightarrow \rightarrow|\mathrm{V},-3 / 2\rangle \rightarrow|c,-1 / 2\rangle$, $|V,-3 / 2\rangle \rightarrow|\mathrm{V},-3 / 2\rangle \rightarrow \quad|\mathrm{V},-1 / 2\rangle \rightarrow|c,-1 / 2\rangle, \quad|V,-3 / 2\rangle \rightarrow|\mathrm{V},-1 / 2\rangle \rightarrow \quad|\mathrm{V},-3 / 2\rangle \rightarrow|c,-1 / 2\rangle$, $|V,-3 / 2\rangle \rightarrow|\mathrm{V},-1 / 2\rangle \rightarrow|\mathrm{V},-1 / 2\rangle \rightarrow|c,-1 / 2\rangle$ we have:

$$
\frac{i}{\sqrt{2}} P_{c} B^{2} k^{2} e_{+}^{\prime}\left\{\frac{1}{(-2 \hbar \omega)}\left[\frac{\left(4\left(\frac{A}{B}-1\right)^{2} e_{z}^{\prime 2}\right)}{(-\hbar \omega)}+\frac{3\left|e_{-}^{\prime}\right|^{2}}{\left(E_{l h}-E_{h h}-\hbar \omega\right)}\right]+\frac{2 e_{z}^{\prime 2}}{\left(E_{l h}-E_{h h}-2 \hbar \omega\right)}\left[\frac{2\left(\frac{A}{B}-1\right)}{(-\hbar \omega)}+\frac{2\left(\frac{A}{B}+1\right)}{\left(E_{l h}-E_{h h}-\hbar \omega\right)}\right]\right\}
$$

TABLE I: EFFECTIVE HAMILTONIAN FOR ELECTRONS IN THE KANE APPROXIMATION

\begin{tabular}{|c|c|c|c|c|c|c|c|}
\hline$E_{c}+\frac{\hbar^{2} k^{2}}{2 m_{c}}$ & 0 & $\frac{i P}{\sqrt{2}} k_{+}$ & $-i \sqrt{\frac{2}{3}} P k_{z}$ & $\frac{-i P}{\sqrt{6}} k_{-}$ & 0 & $\frac{-i P}{\sqrt{3}} k_{z}$ & $\frac{-i P}{\sqrt{3}} k_{-}$ \\
\hline 0 & $E_{c}+\frac{\hbar^{2} k^{2}}{2 m_{c}}$ & 0 & $\frac{i P}{\sqrt{6}} k_{+}$ & $-i \sqrt{\frac{2}{3}} P k_{z}$ & $\frac{-i P}{\sqrt{2}} k_{-}$ & $\frac{-i P}{\sqrt{3}} k_{+}$ & $\frac{i P}{\sqrt{3}} k_{z}$ \\
\hline$\frac{-i P}{\sqrt{2}} k_{-}$ & 0 & $F$ & $H$ & $I$ & 0 & $\frac{H}{\sqrt{2}}$ & $\sqrt{2} I$ \\
\hline$i \sqrt{\frac{2}{3}} P k_{z}$ & $\frac{-i P}{\sqrt{6}} k_{-}$ & $H^{*}$ & $G$ & 0 & $I$ & $\frac{G-F}{\sqrt{2}}$ & $-\sqrt{\frac{3}{2}} H$ \\
\hline$\frac{i P}{\sqrt{6}} k_{+}$ & $i \sqrt{\frac{2}{3}} P k_{z}$ & $I^{*}$ & 0 & $G$ & $-H$ & $-\sqrt{\frac{3}{2}} H^{*}$ & $\frac{F-G}{\sqrt{2}}$ \\
\hline 0 & $\frac{i P}{\sqrt{2}} k_{+}$ & 0 & $I^{*}$ & $-H^{*}$ & $F$ & $-\sqrt{2} I^{*}$ & $-\frac{H^{*}}{\sqrt{2}}$ \\
\hline$\frac{i P}{\sqrt{3}} k_{z}$ & $\frac{i P}{\sqrt{3}} k_{-}$ & $\frac{H^{*}}{\sqrt{2}}$ & $\frac{G-F}{\sqrt{2}}$ & $-\sqrt{\frac{3}{2}} H$ & $-\sqrt{2} I$ & $\frac{F+G}{2}-\Delta$ & 0 \\
\hline$\frac{i P}{\sqrt{3}} k_{+}$ & $-\frac{i P}{\sqrt{3}} k_{z}$ & $\sqrt{2} I^{*}$ & $-\sqrt{\frac{3}{2}} H^{*}$ & $\frac{F-G}{\sqrt{2}}$ & $\frac{H}{\sqrt{2}}$ & 0 & $\frac{F+G}{2}-\Delta$ \\
\hline
\end{tabular}

Then the spectral dependences of the coefficients of the linear-circular dichroism of these optical transitions, determined using the probabilities of these transitions, are shown in Fig. 1. Fig. 1 shows that the spectral dependence of the linear-circular dichroism coefficient $(\eta(\omega))$ depends on the type of optical transitions. In particular, for the first type of optical transition, it increases with an increase in the frequency of light, and for the second type of optical transition, with an increase in the frequency of light, it first decreases and, passing through the minimum, increases. 


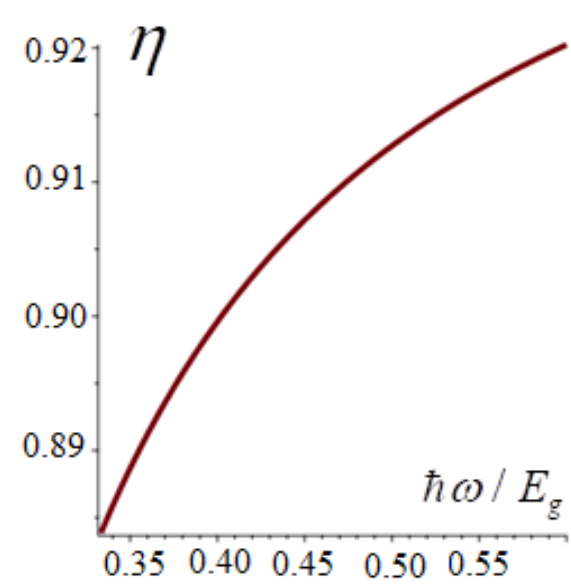

a)

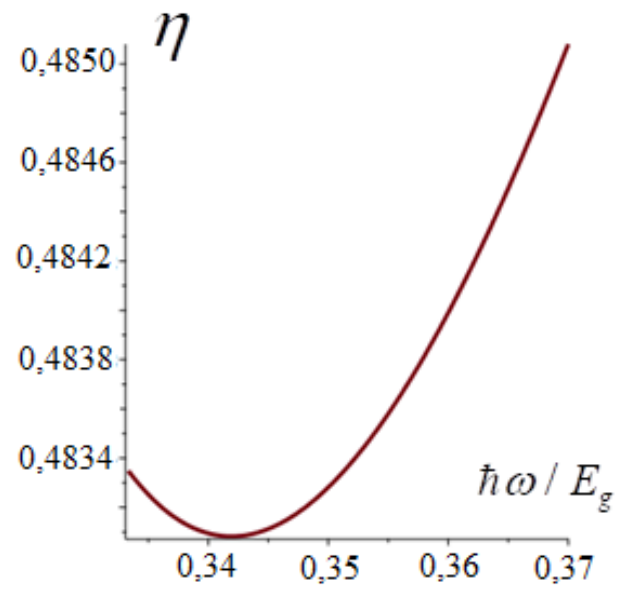

b)

Fig. 1. Spectral dependence of the linear-circular dichroism coefficient for three-photon interband light absorption in crystals of cubic symmetry for two cases.

\section{MULTIPHOTON INTERBAND ABSORPTION OF LIGHT}

The coefficient of interband three-photon light absorption is calculated as:

$$
K_{\text {summ }}^{(N)}(\omega, T)=\sum_{c, m_{c}^{\prime} ; \mathrm{V}, m_{V}^{\prime}} K_{c, m_{c}^{\prime} ; \mathrm{V}, m_{V}^{\prime}}^{(N)}(\omega, T)=\frac{N \hbar \omega}{I} \sum_{c, m_{c}^{\prime} ; \xi, m_{\varsigma}^{\prime} ; \mathrm{V}, m_{V}^{\prime}} W_{c, m_{c}^{\prime} ; \varsigma, m_{\varsigma}^{\prime} ; \mathrm{V}, m_{V}^{\prime}}^{(N)} .
$$

Here $K_{c, m_{c}}^{(N)} ; \mathrm{V}, m_{V}(\omega, T)$ is partial interband three-photon absorption coefficient of light at which when calculating it is necessary sum over the intermediate states (at a fixed $\left(\left|\varsigma, m_{\varsigma}^{\prime}\right\rangle\right)$ and final states $\left(\left|c, m_{c}^{\prime}\right\rangle\left(m_{c}^{\prime} \pm 1 / 2\right)\right) ; K_{\text {summ }}^{(N)}(\omega, T)$ is total three-photon absorption coefficient of light, $W_{c, m_{c}^{\prime} ; \varsigma, m_{\varsigma}^{\prime} ; \mathrm{V}, m_{V}^{\prime}}^{(N)}$ is the probability of the optical transition between the valence band and the conduction band, defined as:

$$
W_{c, m_{c} ; \varsigma, m_{\varsigma} ; \mathrm{V}, m_{V}}^{(N)}=\frac{2 \pi}{\hbar} \sum_{\varsigma, m_{\varsigma}}\left|M_{c, m_{c} ; \varsigma, m_{\varsigma} ; \mathrm{V}, m_{V}}^{(N)}(\boldsymbol{k})\right|^{2} \cdot\left[f_{c}(\boldsymbol{k})-f_{V_{l}}(\boldsymbol{k})\right] \cdot \delta\left(E_{c}(\boldsymbol{k})-E_{V_{l}}(\boldsymbol{k})-N \hbar \omega\right),
$$

where $\left|\varsigma, m_{\varsigma}^{\prime}\right\rangle$ is the virtual state can be located both in the conduction band and in the subband of heavy holes $\left|\mathrm{V}_{h h}, m_{h h}^{\prime}\right\rangle\left(m_{h h}^{\prime}= \pm 3 / 2\right)$ or in the subband of light holes $\left|\mathrm{V}_{l h}, m_{l h}^{\prime}\right\rangle\left(m_{l h}^{\prime}= \pm 1 / 2\right)$, as well as in the spin-split subband $\left|S O, m_{S O}^{\prime}\right\rangle\left(m_{S O}^{\prime}= \pm 1 / 2\right)$ of the valence band, $M_{c, m_{c}^{\prime} ; \zeta, m_{\zeta}^{\prime} ; \mathrm{V}, m_{V}^{\prime}}^{(N)}(\boldsymbol{k})$ is the composite matrix element of the optical transition under consideration, $f_{c}(\boldsymbol{k})\left[f_{V_{l}}(\boldsymbol{k})\right]$ are the distribution functions of current carriers, $E_{c}(\boldsymbol{k})\left[E_{V_{l}}(\boldsymbol{k})\right]$ is the energy spectrum of electrons (holes), is the eigenvalue of the total angular momentum operator in the band with number $v\left(c, V_{l}, S O\right)$ . The matrix element of the electron-photon interaction is defined as $H_{l l^{\prime}}=\frac{e}{i m_{0} \omega}\left(\frac{2 \pi I}{n_{\omega} c}\right)^{1 / 2}(\vec{e} \cdot \vec{p})_{l l^{\prime}}$, where $\vec{p}$ is the operator is the momentum, $\vec{A}$ is the vector potential of the electromagnetic wave, $I$ is the intensity of light, and $n_{\omega}$ is the refractive index of the medium at the frequency $\omega$.

Since the probability of a multiphoton optical transition, which is used to determine the coefficient of multiphoton light absorption, is expressed as:

$$
\begin{aligned}
& W_{c, m_{c}^{\prime} ; \mathrm{V}, m_{V}^{\prime}}^{(N)}=\frac{1}{\pi \hbar}\left(\frac{e}{m_{0} \omega}\right)^{2 N}\left(\frac{2 \pi I}{n c}\right)^{N}\left(k_{c, m_{V_{l}}}^{(N \omega)}\right)^{3}\left(N \hbar \omega-E_{\mathrm{g}}\right)^{-1}\left|\mathfrak{R}_{c, m_{c}^{\prime} ; \mathrm{V}, m_{V}^{\prime}}^{(N)}\left(k_{c, m_{V_{l}}}^{(N \omega)}\right)\right|^{2} \times \\
& {\left[f\left(E_{c}\left(k_{c, m_{V_{l}}}^{(N \omega)}\right)\right)-f\left(E_{V_{l}}\left(k_{c, m_{V_{l}}}^{(N \omega)}\right)\right)\right]}
\end{aligned}
$$


where $k_{c, m_{V_{l}}}^{(N \omega)}=\left[\frac{2 m_{c} m_{V_{l}}}{m_{c}+m_{V_{l}}}\right]\left(N \hbar \omega-E_{g}\right), m_{c}\left(m_{V_{l}}\right)$ is effective mass in the zone $c\left(\mathrm{~V}_{l}\right),\left|\mathfrak{R}_{c, m_{c}^{\prime} ; s, m_{\varsigma}^{\prime} ; \mathrm{V}, m_{V}^{\prime}}^{(N)}\left(k_{c, m_{V_{l}}}^{(N \omega)}\right)\right|^{2}$ the quantity determined by the integral of the type $\int_{-1}^{1} d \cos (\theta) \int_{0}^{2 \pi} d \varphi \int_{0}^{\infty} k d k^{2} \sum_{\zeta, m_{\zeta}}\left|M_{c, m_{c}^{\prime} ; \zeta, m_{\varsigma}^{\prime} ; \mathrm{V}, m_{V}^{\prime}}^{(N)}(k, \theta, \varphi)\right|^{2}, f_{c}\left(k_{c, m_{h h}}^{(3 \omega)}\right)\left[f_{h h}\left(k_{c, m_{h h}}^{(3 \omega)}\right)\right]$ is the distribution function of electrons (holes with energy $E_{c}\left(k_{c, m_{h h}}^{(3 \omega)}\right)\left[E_{h h}\left(k_{c, m_{h h}}^{(3 \omega)}\right)\right]$ ). Let us consider the specific case. Let $M_{c, m_{c}^{\prime} ; \zeta, m_{\varsigma}^{\prime} ; \mathrm{V}, m_{V}^{\prime}}^{(N)}\left(k_{c, m_{V_{l}}}^{(N \omega)}\right) \quad$ is the composite matrix element consist of two terms, i.e. $M_{c, m_{c}^{\prime} ; \zeta, m_{\zeta}^{\prime} ; \mathrm{V}, m_{V}^{\prime}}^{(N)}\left(k_{c, m_{V_{l}}}^{(N \omega)}\right)=M_{c, m_{c}^{\prime} ; \zeta, m_{\varsigma}^{\prime} ; \mathrm{V}, m_{V}^{\prime}}^{(A)}\left(k_{c, m_{V_{l}}}^{(N \omega)}\right)+M_{c, m_{c}^{\prime} ; \zeta, m_{\xi}^{\prime} ; \mathrm{V}, m_{V}^{\prime}}^{(B)}\left(k_{c, m_{V_{l}}}^{(N \omega)}\right)$, where $M_{c, m_{c}^{\prime} ; \zeta, m_{\zeta}^{\prime} ; \mathrm{V}, m_{V}^{\prime}}^{(A)}\left(k_{c, m_{V_{l}}}^{(N \omega)}\right)$ is the matrix element of the optical transition of the type " $A$ ", and $M_{c, m_{c}^{\prime} ; \zeta, m_{\varsigma}^{\prime} ; \mathrm{V}, m_{V}^{\prime}}^{(B)}\left(k_{c, m_{V_{l}}}^{(N \omega)}\right)$ is the matrix element of the optical transition of the type " $B$ ":

$$
\begin{aligned}
& \left|M_{c, m_{c}^{\prime} ; \varsigma, m_{\varsigma}^{\prime} ; \mathrm{V}, m_{V}^{\prime}}^{(N)}\left(k_{c, m_{V_{l}}}^{(N \omega)}\right)\right|^{2}=\left|M_{c, m_{c}^{\prime} ; \varsigma, m_{\zeta}^{\prime} ; \mathrm{V}, m_{V}^{\prime}}^{(A)}\left(k_{c, m_{V_{l}}}^{(N \omega)}\right)+M_{c, m_{c}^{\prime} ; \varsigma, m_{\varsigma}^{\prime} ; \mathrm{V}, m_{V}^{\prime}}^{(B)}\left(k_{c, m_{V_{l}}}^{(N \omega)}\right)\right|^{2}= \\
& =\mid M_{c, m_{c}^{\prime} ; \zeta, m_{\varsigma}^{\prime} ; \mathrm{V}, m_{V}^{\prime}}^{(A)}\left(\left.k_{c, m_{V_{l}}}^{(N \omega)}\right|^{2}+\left|M_{c, m_{c}^{\prime} ;, m_{\varsigma}^{\prime} ; \mathrm{V}, m_{V}^{\prime}}^{(B)}\left(k_{c, m_{V_{l}}}^{(N \omega)}\right)\right|^{2}+\left[M_{c, m_{c}^{\prime} ; \zeta, m_{\varsigma}^{\prime} ; \mathrm{V}, m_{V}^{\prime}}^{(A)}\left(k_{c, m_{V_{l}}}^{(N \omega)}\right)\right]^{*} M_{c, m_{c}^{\prime} ; \zeta, m_{\varsigma}^{\prime} ; \mathrm{V}, m_{V}^{\prime}}^{(B)}\left(k_{c, m_{V_{l}}}^{(N \omega)}\right)+\right. \\
& M_{c, m_{c}^{\prime} ; \zeta, m_{\zeta}^{\prime} ; \mathrm{V}, m_{V}^{\prime}}^{(A)}\left(k_{c, m_{V_{l}}}^{(N \omega)}\right)\left[M_{c, m_{c}^{\prime} ; \zeta, m_{\zeta}^{\prime} ; \mathrm{V}, m_{V}^{\prime}}^{(B)}\left(k_{c, m_{V_{l}}}^{(N \omega)}\right)\right]^{*} \text {. }
\end{aligned}
$$

Whence we have that the total light absorption coefficient consists of the sum of the partial light absorption coefficients, determined by the quantities $\left|M_{c, m_{c}^{\prime} ; s, m_{\varsigma}^{\prime} ; \mathrm{V}, m_{V}^{\prime}}^{(A)}\left(k_{c, m_{V_{l}}}^{(N \omega)}\right)\right|^{2}$ and $\left|M_{c, m_{c}^{\prime} ; s, m_{\varsigma}^{\prime} ; \mathrm{V}, m_{V}^{\prime}}^{(B)}\left(k_{c, m_{V_{l}}}^{(N \omega)}\right)\right|^{2}$, as well as the contributions to the total light absorption coefficient, determined by the quantity $\left[M_{c, m_{c}^{\prime} ; s, m_{\xi}^{\prime} ; \mathrm{V}, m_{V}^{\prime}}^{(A)}\left(k_{c, m_{V_{l}}}^{(N \omega)}\right)\right]^{*} M_{c, m_{c}^{\prime} ; s, m_{\zeta}^{\prime} ; \mathrm{V}, m_{V}^{\prime}}^{(B)}\left(k_{c, m_{V_{l}}}^{(N \omega)}\right)$, i.e.

$$
K_{c, m_{c}^{\prime} ; \mathrm{V}, m_{V}^{\prime}}^{(\mathrm{A}, \mathrm{B}, \mathrm{C})}(\omega, T)=\frac{N \hbar \omega}{I} W_{c, m_{c}^{\prime} ; \zeta, m_{\varsigma}^{\prime} ; \mathrm{V}, m_{V}^{\prime}}^{(A, \mathrm{~B}, \mathrm{C})}
$$

where

$$
\begin{aligned}
& W_{c, m_{c}^{\prime}, \mathrm{V}, m_{V}^{\prime}}^{(\mathrm{A}, B)}=\frac{1}{\pi \hbar}\left(\frac{e}{m_{0} \omega}\right)^{2 N}\left(\frac{2 \pi I}{n c}\right)^{N}\left(k_{c, m_{V_{l}}}^{(N \omega)}\right)^{3}\left(N \hbar \omega-E_{\mathrm{g}}\right)^{-1}\left|M_{c, m_{c}^{\prime} ; \mathrm{V}, m_{V}^{\prime}}^{(A, B)}\left(k_{c, m_{V_{l}}}^{(N \omega)}\right)\right|^{2} \times \\
& {\left[f\left(E_{c}\left(k_{c, m_{V_{l}}}^{(N \omega)}\right)\right)-f\left(E_{V_{l}}\left(k_{c, m_{V_{l}}}^{(N \omega)}\right)\right)\right] \text {, }} \\
& W_{c, m_{c}^{\prime}: \mathrm{V}, m_{V}^{\prime}}^{(\mathrm{C})}=\frac{1}{\pi \hbar}\left(\frac{e}{m_{0} \omega}\right)^{2 N}\left(\frac{2 \pi I}{n c}\right)^{N}\left(k_{c, m_{V_{l}}}^{(N \omega)}\right)^{3}\left(N \hbar \omega-E_{\mathrm{g}}\right)^{-1}\left[f\left(E_{c}\left(k_{c, m_{V_{l}}}^{(N \omega)}\right)\right)-f\left(E_{V_{l}}\left(k_{c, m_{V_{l}}}^{(N \omega)}\right)\right)\right] \times \\
& \times\left|\left[M_{c, m_{c}^{\prime} ; s, m_{\zeta}^{\prime} ; \mathrm{V}, m_{V}^{\prime}}^{(A)}\left(k_{c, m_{V_{l}}}^{(N \omega)}\right)\right]^{*} M_{c, m_{c}^{\prime} ; s, m_{\zeta}^{\prime} ; \mathrm{V}, m_{V}^{\prime}}^{(B)}\left(k_{c, m_{V_{l}}}^{(N \omega)}\right)+M_{c, m_{c}^{\prime} ; s, m_{\zeta}^{\prime} ; \mathrm{V}, m_{V}^{\prime}}^{(A)}\left(k_{c, m_{V_{l}}}^{(N \omega)}\right)\left[M_{c, m_{c}^{\prime} ; s, m_{\zeta}^{\prime} ; \mathrm{V}, m_{V}^{\prime}}^{(B)}\left(k_{c, m_{V_{l}}}^{(N \omega)}\right)\right]^{*}\right|^{2},
\end{aligned}
$$

calculating them separately, one can consider that the numerical contribution of the partial absorption coefficients of light. Therefore, we will consider specific optical transitions. For example, for an optical transition of the type $|V,-3 / 2\rangle \Rightarrow$ $\Rightarrow|\mathrm{V},-3 / 2\rangle \rightarrow|c,-1 / 2\rangle, \quad|V,-3 / 2\rangle \Rightarrow|\mathrm{V},+1 / 2\rangle \rightarrow|c,-1 / 2\rangle, \quad|V,-3 / 2\rangle \Rightarrow|\mathrm{V},-1 / 2\rangle \rightarrow|c,-1 / 2\rangle$, the coefficient of three-photon interband absorption of light for crystals of cubic symmetry is determined by the relation

$$
K_{c, h h, 1}^{(N=3)}=\frac{3}{4} K_{g}^{(3)}(0) \frac{m_{c} m_{h h}}{\left(m_{c}+m_{h h}\right) m_{0}} I^{2} \frac{E_{g}^{2}}{B^{2} k_{g}^{4}}\left[f_{c}\left(k_{c, m_{h h}}^{(3 \omega)}\right)-f_{h h}\left(k_{c, m_{h h}}^{(3 \omega)}\right)\right] x_{\omega}^{-5} r_{c, m_{h h}}^{(3 \omega)} \times
$$




$$
\left[\left(\frac{2\left(\frac{A}{B}-1\right)}{x_{\omega}}\right)^{2} a_{c, h h, 1}^{(N=3)}+2 \frac{2\left(\frac{A}{B}-1\right)}{-x_{\omega}} \frac{b_{c, h h, 1}^{(N=3)}}{x_{l h}-x_{h h}-2 x_{\omega}}+\frac{c_{c, h h, 1}^{(N=3)}}{\left(x_{l h}-x_{h h}-2 x_{\omega}\right)^{2}}\right],
$$

and for transitions of type $\quad|V,-3 / 2\rangle \rightarrow|\mathrm{V},-3 / 2\rangle \rightarrow|\mathrm{V},-3 / 2\rangle \rightarrow|c,-1 / 2\rangle, \quad|V,-3 / 2\rangle \rightarrow|\mathrm{V},-3 / 2\rangle \rightarrow$ $|\mathrm{V},-1 / 2\rangle \rightarrow|c,-1 / 2\rangle,|V,-3 / 2\rangle \rightarrow|\mathrm{V},-1 / 2\rangle \rightarrow|\mathrm{V},-3 / 2\rangle \rightarrow|c,-1 / 2\rangle, \quad|V,-3 / 2\rangle \rightarrow|\mathrm{V},-1 / 2\rangle \rightarrow|\mathrm{V},-1 / 2\rangle \rightarrow|c,-1 / 2\rangle \quad$ we have:

$$
\begin{aligned}
& K_{c, h h, 2}^{(N=3)}=6 K_{g}^{(3)}(0) \frac{m_{c} m_{h h}}{\left(m_{c}+m_{h h}\right) m_{0}} I^{2}\left[f_{c}\left(k_{c, m_{h h}}^{(3 \omega)}\right)-f_{h h}\left(k_{c, m_{h h}}^{(3 \omega)}\right)\right]\left(x_{\omega}^{-5}\right)\left(r_{c, m_{h h}}^{(3 \omega)}\right)^{5}\left(a_{c, h h, 2}^{(N=3)} \mathfrak{R}_{1}^{2}+b_{c, h h, 2}^{(N=3)} \mathfrak{R}_{1} \mathfrak{R}_{2}+c_{c, h h, 2}^{(N=3)} \mathfrak{R}_{2}^{2}\right) E_{g}^{4} \\
& r_{c, m_{h h}}^{(3 \omega)}=k_{c, m_{h h}}^{(3 \omega)} /
\end{aligned}
$$

where $K_{g}^{(3)}(0)=\pi m_{0}\left(\frac{2 \pi e^{2}}{n_{\omega} c h}\right)^{3} k_{\mathrm{g}}^{5} E_{\mathrm{g}}^{-9} P_{c}^{2} B^{4}, x_{\omega}=\hbar \omega / E_{g}, k_{c, m_{V_{l}}}^{(3 \omega)}=\frac{2 m_{c} m_{V_{l}}}{m_{c}+m_{V_{l}}} \frac{3 \hbar \omega-E_{g}}{\hbar^{2}}, x_{l}=E_{l}\left(k_{\omega}\right) / E_{g}, l=l h, h h, S O$, $r_{c, m_{V_{l}}}^{(N \omega)}=\frac{2 m_{c} m_{V_{l}}}{m_{c}+m_{V_{l}}} \frac{N \hbar \omega-E_{g}}{m_{0} E_{g}}, k_{\omega}$ - is wave vector of current carriers, determined by the energy conservation law $E_{l h}(k)-E_{h h}(k)-3 \hbar \omega=0, \quad$ for $\quad$ linearly polarized light $\quad a_{c, h h, 1}^{(N=3)}=\frac{90}{135}, b_{c, h h, 1}^{(N=3)}=\frac{180}{135}, c_{c, h h, 1}^{(N=3)}=\frac{488}{135}, \quad a_{c, h h, 2}^{(N=3)}=\frac{1}{15}$, $b_{c, h h, 2}^{(N=3)}=c_{c, h h, 2}^{(N=3)}=\frac{4}{27}$ for circularly polarized light $a_{c, h h, 1}^{(N=3)}=\frac{70}{105}, b_{c, h h, 1}^{(N=3)}=\frac{133}{105}, c_{c, h h, 1}^{(N=3)}=\frac{396}{105}, \quad a_{c, h h, 2}^{(N=3)}=\frac{8}{105}, b_{c, h h, 2}^{(N=3)}=\frac{13}{105}$, $c_{c, h h, 2}^{(N=3)}=\frac{18}{105}, \Re_{1}=\frac{2\left(\frac{A}{B}-1\right)^{2}}{(\hbar \omega)^{2}}-\frac{2\left(\frac{A}{B}-1\right)}{\left(E_{l h}-E_{h h}-2 \hbar \omega\right)(\hbar \omega)}+\frac{\left.\frac{A}{B}+1\right)}{\left(E_{l h}-E_{h h}-2 \hbar \omega\right)\left(E_{l h}-E_{h h}-\hbar \omega\right)}, \Re_{2}=-\frac{3}{2 \hbar \omega\left(E_{l h}-E_{h h}-\hbar \omega\right)}$.

\section{ANALYSIS OF THE RESULTS}

The spectral dependences of the coefficients of three-photon interband absorption of light for the above optical transitions are shown in Fig. 2. It can be seen from Fig. 1 that the spectral dependence of $K_{c, h h, 2}^{(N=3)} / K_{g}^{(3)}(0)$ depends on the type of optical transitions. In particular, for the first and second types of optical transitions, with an increase in the frequency of light, it increases and passing through a maximum decrease for both linearly polarized light and circularly polarized light, but the maxima of the dependence differ from each other in value. The numerical values of the band parameters of InSb were taken from [9].
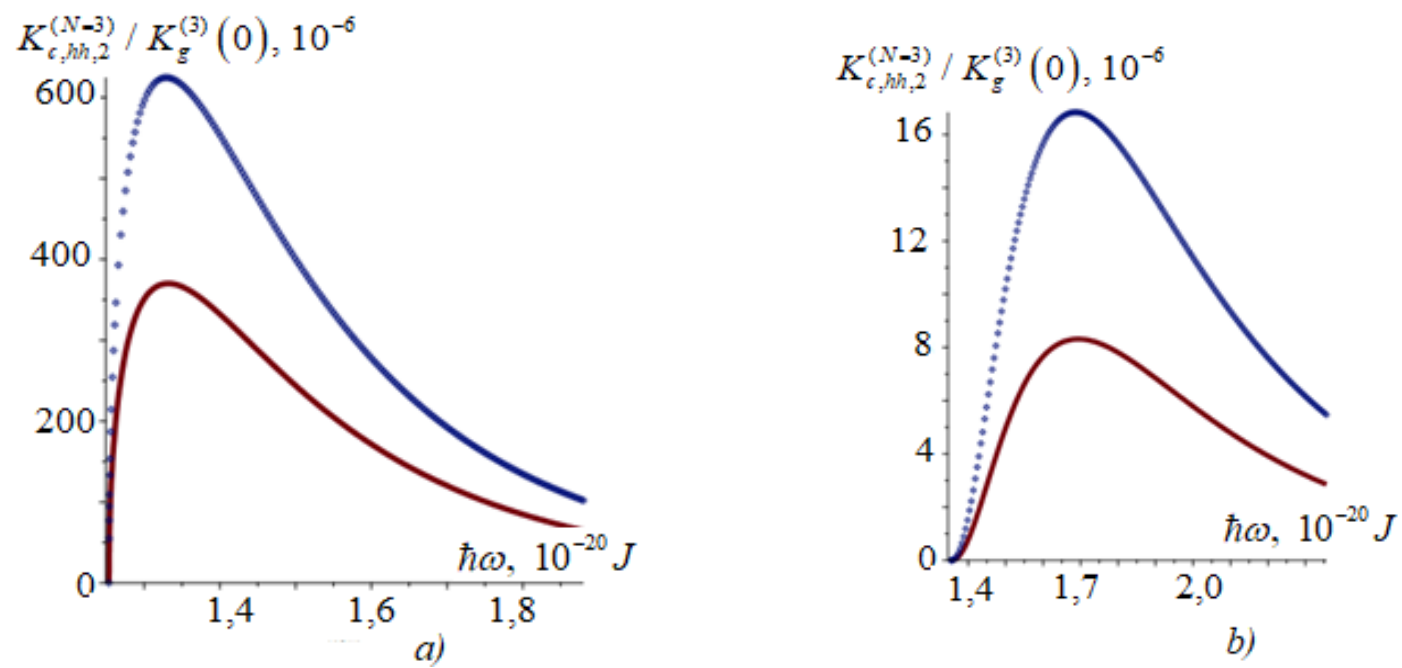

Fig. 2. Spectral dependence of the coefficient of three-photon interband light absorption in InSb crystals for the two cases considered in the text. 


\section{REFERENCES}

[1] A. Miller, A. Johnston, J. Dempsey, J. Smith, C. R. Pidgeon, and G. D. Holah, “Two-photon absorption in InSb and Hg I-x Cd $_{x}$ Te," J. Phys., 1978, vol. 12, no. 22, pp. 4839-4849. https://iopscience.iop.org/article/10.1088/0022-3719/12/22/025.

[2] C. R. Pidgeon, B. S Wherrett, A. M. Johnston, J. Dempsey, A. Miller, "Two-photon absorption in zinc-blende semiconductors," Phys. Rev. Lett., 1979, vol. 42, no. 24, pp. 1785-1788, and references therein. DOI:https://doi.org/10.1103/PhysRevLett.43.1843.2.

[3] R. Braunstein, N. Ockman, "Optical double-photon absorption in CdS," Phys. Rev. A., 1964, vol. 34, pp. 499-507.

[4] E. L. Ivchenko, "Two-photon absorption and optical orientation of free carriers in cubic crystals," Semiconductors, 1972, vol. 14, no. 12, pp. 3489-3485. (in Russian).

[5] S. B. Arifzhanov, E. L. Ivchenko, "Multiphoton light absorption in crystals with a diamond and zinc blende structure," Physics of the Solid State, 1975, vol. 17, no. 1, pp. $81-89$ (in Russian).

[6] R. Ya. Rasulov, "Linear circular dichroism in multiphoton interband absorption in semiconductors," Physics of the Solid State, 1993, vol. 35, no. 6, pp. 1674-1678 (in Russian).

[7] V. R. Rasulov, R. Ya. Rasulov, I. Eshboltaev, "Linear-Circular Dichroism of Four-Photon Absorption of Light in Semiconductors with a Complex Valence Band," Physics of the Solid State, 2017, vol. 59, no. 3, pp. 463-468. DOI: 10.1134/S1063783417030283.

[8] E. L. Ivchenko, R. Ya. Rasulov, "Symmetry and real band structure of semiconductors," Uzbekistan. Tashkent. Fan., 1989. 126 p (in Russian).

[9] I. Vurgaftman, J. R. Meyer, L. R. Ram-Mohan, "Band parameters for III-V compound semiconductors and their alloys," Journal of Applied Physics, 2001, vol. 89, no. 11, pp. 5815-5875. https://doi.org/10.1063/1.1368156. 